\title{
Ultrasonography-guided Suction Thrombectomy for an Isolated Portal Vein Thrombus in Liver Surgery
}

\author{
YO-ICHI YAMASHITA, KATSUNORI IMAI, SHIGEKI NAKAGAWA, YOSUKE NAKAO, TOSHIHIKO YUSA, \\ RUMI ITOYAMA, HIROHISA OKABE, HIROMITSU HAYASHI, AKIRA CHIKAMOTO and HIDEO BABA \\ Department of Gastroenterological Surgery, Graduate School of Medical Sciences, \\ Kumamoto University, Kumamoto, Japan
}

\begin{abstract}
Background: Removal of an isolated portal vein $(P V)$ thrombus is still a difficult problem in liver surgery. In this report, we present technical details of the ultrasonography (US)-guided suction thrombectomy for an isolated $P V$ thrombus in a case with PV tumor thrombus extending into the main PV trunk of a patient undergoing right hemihepatectomy and tumor thrombectomy for hepatocellular carcinoma (HCC). Patients and Methods: Three cases were treated with US-guided suction thrombectomy for an isolated $P V$ thrombus in liver surgeries. All three patients had HCC; however, two cases had a blood clot PV thrombus, and one case had an isolated PV tumor thrombus. Results: In all three cases, the isolated PV thrombus was successfully removed by suction thrombectomy without intraoperative and postoperative problems. Conclusion: US-guided suction thrombectomy is a promising method for removal of an isolated PV thrombus in liver surgery.
\end{abstract}

Portal venous (PV) tumor thrombus is one of the worst prognostic factors in patients with hepatocellular carcinoma (HCC); however, the efficacy of curative resection has been noted to achieve acceptable results such as a $40 \% 5$-year survival rate (1). To remove a PV tumor thrombus extending into the main portal trunk or the contralateral portal vein, we usually use not the en bloc technique (2) but the back-flow (3) or peeling-off techniques (1). After performing the peeling-off technique, on rare occasions, an isolated PV

This article is freely accessible online.

Correspondence to: Yo-ichi Yamashita, MD, Ph.D., F.A.C.S., Department of Gastroenterological Surgery, Graduate School of Medical Sciences, Kumamoto University 1-1-1 Honjo, Chuo-ku, Kumamoto 860-8556, Japan. Tel: +81 963735211 Fax: +81 963714378, e-mail: y-yama@kumamoto-u.ac.jp

Key Words: Suction thrombectomy, portal vein thrombus, liver surgery. tumor thrombus in the remnant liver may be encountered, and removal of this isolated PV tumor thrombus is sometimes troublesome. We reported that a blood clot PV thrombus is a relatively important problem in patients undergoing liver surgeries $(4,5)$. Checking for and removing any small blood clot PV thrombus intraoperatively are thus very important procedures. We present the technical details of ultrasonography (US)-guided suction thrombectomy for an isolated PV thrombus in liver surgery, and initial clinical results.

\section{Patients and Methods}

We have experienced three cases with US-guided suction thrombectomy for an isolated PV thrombus in liver surgeries, and summarize their clinical characteristics and outcomes in Table I. Case 1 is presented in this report.

A 51-year-old male had a huge HCC $(10 \mathrm{~cm}$ in diameter $)$ located in segment 8 of the liver with a PV tumor thrombus extending into the main portal trunk and the first branch of the left PV (Figure 1). He underwent right hemi-hepatectomy with tumor thrombectomy by the peeling-off technique. The main trunk of the PV and the first branch of the left and right PVs were isolated and controlled with tapes. To avoid the escape of stray PV tumor thrombus into the peripheral left PV, the peripheral side of the left PV was clamped using bulldog forceps. After the clamping of the proximal side of the main PV using Potts forceps, the first branch of the right PV was cut in a circle using Metzenbaum surgical scissors; the PV tumor thrombus was excised by the peeling-off technique. To remove the isolated PV tumor thrombus, the PV was flushed by declamping the Potts forceps. The main trunk and the left branch of the PV at the range from the hole of the right PV to both forceps were irrigated using a saline mixture including heparin, then the hole in the right $\mathrm{PV}$ was closed by running suture using 5-0 Proline ${ }^{\circledR}$ (ETHICON, Inc.). After completion of the right hemihepatectomy, we found a small isolated PV tumor thrombus at the umbilical portion by intraoperative US (XarioXG; Toshiba, Inc.) (Figure 2). We performed US-guided suction thrombectomy for this isolated PV tumor thrombus. After taping the main trunk and the left branch of the PV, the suture of 5-0 Proline at the stump of the right PV was cut by Metzenbaum surgical scissors. Back flow from the left branch of the PV was recognized, but the remnant PV thrombus was not excised. A Yankauer suction tip was inserted into 
Table I. Clinical characteristics of three cases with ultrasonography-guided suction thrombectomy for isolated portal vein thrombus.

\begin{tabular}{llcccc}
\hline Case & Diagnosis & Procedure & Location & Pathology & Outcome \\
\hline 1 & HCC (Vp 4) & Right hemi. & UP & Tumor (HCC) & Success \\
2 & HCC (Vp 0) & Right hemi. & UP & Blood clot & Success \\
3 & HCC (Vp 2) & Posterior seg. & Anterior & Blood clot & Success \\
\hline
\end{tabular}

HCC: Hepatocellular carcinoma, Vp: macroscopic portal venous tumor thrombus, hemi: hemihepatectomy, seg: segmentectomy of the liver, UP: umbilical portion.

A

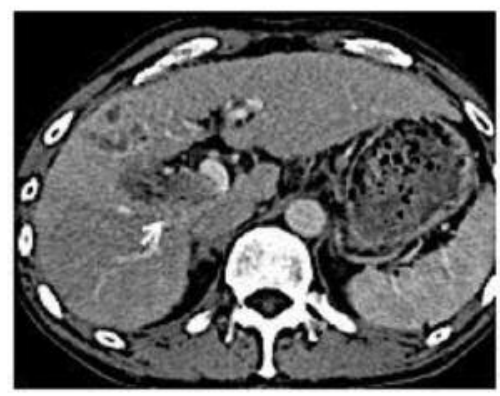

B

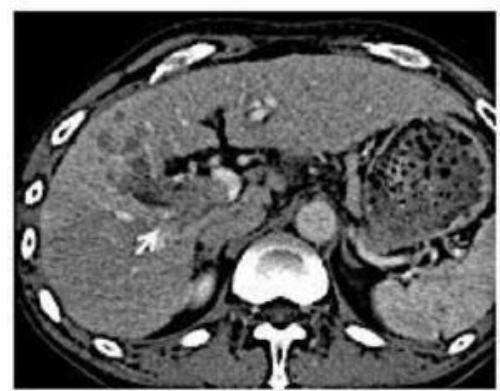

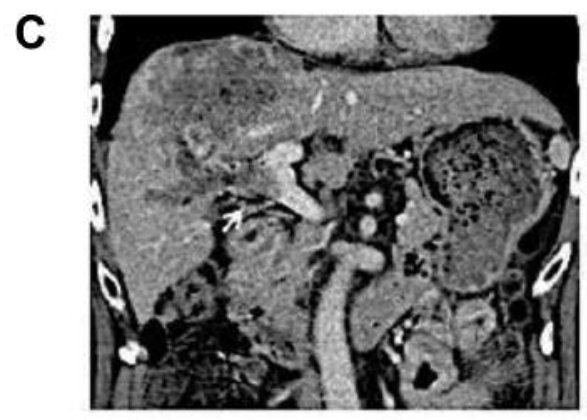

D

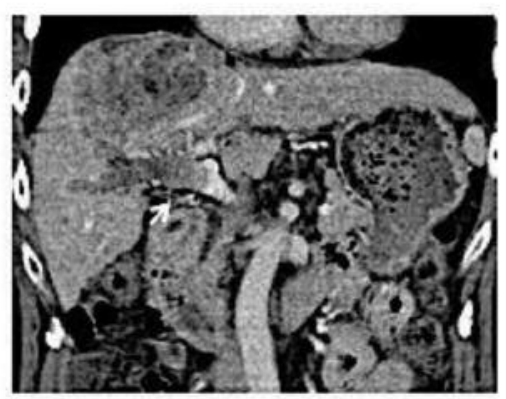

Figure 1. Case 1: Portal vein tumor thrombus of a huge hepatocellular carcinoma extended from the anterior branch of the PV (A: coronary view, $C$ : sagittal view) into the main trunk of the PV (B: coronary view, D: sagittal view).

the left PV from the hole of the right PV, and located near the isolated PV tumor thrombus at the umbilical portion under the US guidance; suction was then switched on. The isolated PV tumor thrombus gradually disappeared by suction. After confirming the absence of the remnant isolated PV tumor thrombus by US, the hole of the stump of the right $\mathrm{PV}$ was closed again by running suture using 5-0 Proline ${ }^{\circledR}$ (ETHICON, Inc.).

\section{Results}

All three patients were diagnosed as having HCC, and two had macroscopic PV tumor thrombus (Vp). According to the Japanese General Rules for the Clinical and Pathological Study of Primary Liver Cancer, Vp4 means that the PV tumor thrombus extends into the main portal trunk or the contralateral portal vein, and Vp2 means that the PV tumor thrombus extends into the second branch of

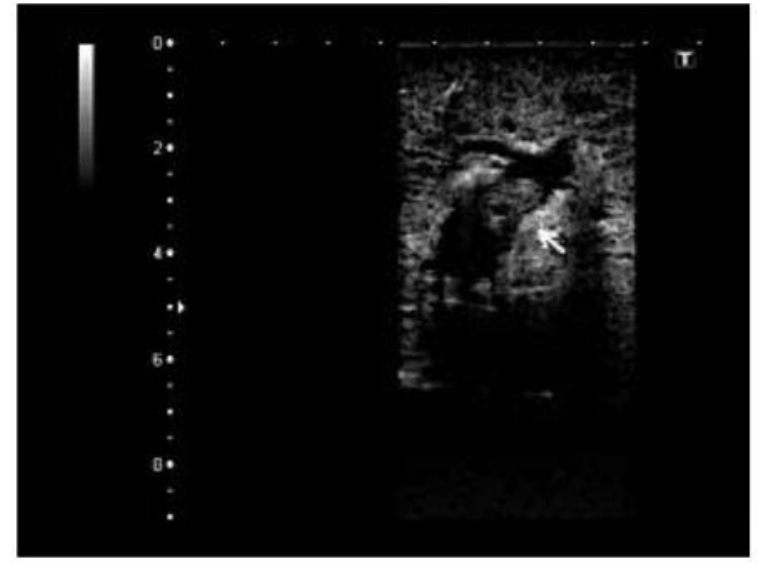

Figure 2. (Case 1) The white arrow indicates an isolated portal vein tumor thrombus at the umbilical portion in intraoperative ultrasonography. 

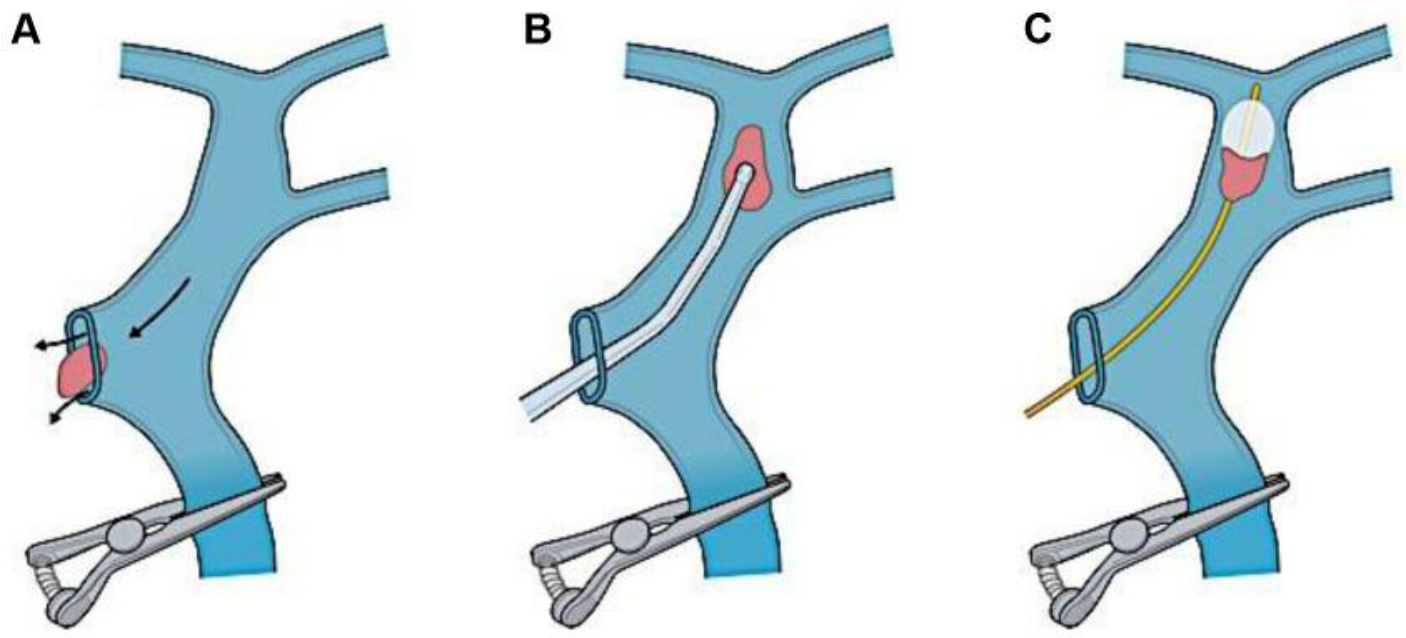

Figure 3. Three possible methods for the removal for an isolated portal vein thrombus: The back-flow technique (A), suction thrombectomy (B), and the Fogarty catheter technique $(C)$.

right or left PVs (Table I) (6). Two cases had a blood clot PV thrombus, and one case had an isolated PV tumor thrombus. We recognized all three isolated PV thrombi after the completion of hepatectomies during the final intraoperative US to check for remnant tumor and visualize blood flow in the remnant liver. Two out of three cases had an isolated PV thrombus at the umbilical portion. A Yankauer suction tip was inserted into the PV from the stump of the right PV in cases 1 and 2, and from the main trunk of the PV in case 3 . In both case 1 and 2, the holes in the PV were made by cutting the existing suture of the stump of the right PV, and in case 3, a new hole in the main trunk of the PV was made. In all three cases, the isolated PV thrombus was successfully removed without major problems. In addition, we paid close attention to avoiding PV stenosis during the suture of the PV hole where the Yankauer tip was inserted. All three patients underwent postoperative anticoagulation therapy with enoxaparin (5).

The long-term outcome of this suction thrombectomy for isolated PV thrombus is another concern. In cases 1 and 2, the enhanced computed tomography (CT) revealed no PV thrombus or PV stricture at the 7th and 90th postoperative days (POD). In case 3, enhanced CT revealed a small PV clot at segment 8 of the liver at 7th POD; however, this small clot had disappeared 30th POD without warfarin use. In case 1, multiple intrahepatic recurrences were identified in enhanced CT 6 months after surgery; however, all three patients remain alive today without problems in PV flow and liver functions ( 1 year in case 1,2 years in case 2 , and 6 months in case 3 ).

\section{Discussion}

Perhaps every liver surgeon removes isolated PV thrombi applying their preferred method, or the method appropriate for the particular case. In Figure 3, the various possible techniques for the removal of an isolated PV thrombus in liver surgery are depicted. The simplest technique may be the so-called back-flow technique (Figure 3A). This method is useful when the distance between the hole of the PV and the isolated PV thrombus is short. In all three of our cases, back-flow was recognized when we made the PV hole; however, no isolated PV thrombus was excised only by back-flow of the PV.

In this report, we present the technique called suction thrombectomy (Figure 3B). It was possible to excise all three isolated PV thrombi successfully by this method. Compared to the back-flow technique, injury to PV endothelial cells poses a possible problem in this method. To avoid this complication, we tried to minimize the contact of the Yankauer tip with the inner membrane of PV and minimized the power and duration of suction. In all three cases, no PV thrombus was found on enhanced CT at 7 and 90 POD.

The final possible method is the Fogarty catheter technique (Figure 3C) (7). This technique is useful when the isolated PV is too hard to be suctioned out. However, an intraoperatively isolated PV thrombus would likely be fresh and not so hard. In addition, this technique introduces the possibility of a fragment of the PV thrombus migrating into a peripheral branch of the PV. In our own experience, we have had no good occasion to use a Fogarty catheter for the removal of a PV thrombus. 
From our experiences, we would present two especially important tips for the use of suction thrombectomy to remove an isolated PV thrombus. Firstly, the timing of the start of suction is important. The proper insertion of a Yankauer into the PV while suction is on is very difficult. The suction should be switched on only after navigating the Yankauer tip close to the isolated PV thrombus. Secondly, the visibility of a Yankauer tip is relatively poor in intraoperative US. In all three of our cases, constant movement of the Yankauer tip helped its visibility in US. We used a plastic Yankauer tip in all three cases. We believe the use of a metallic Yankauer tip might improve its visibility in intraoperative US.

We should also refer to the management of acute PV thrombosis, including conservative management, anticoagulation, and thrombolysis/thrombectomy (8). In recent years, the techniques of endovascular thrombus fragmentation and thrombectomy have rapidly progressed, and these would be alternative options for removal of an intraoperatively isolated PV thrombus (9).

Of course, the clinical utility of suction thrombectomy for isolated PV thrombus should be confirmed in a larger number of patients; however, cases with intraoperatively identified isolated PV thrombus in liver surgery are extremely rare. Therefore, comparisons of clinical efficacies of suction thrombectomy for PV thrombus among patients who either did not undergo suction thrombectomy or who had alternative thrombectomy are difficult and this remains to be investigated.

\section{Conclusion}

US-guided suction thrombectomy appears to be a feasible and useful method for the removal of an isolated PV thrombus in liver surgery.

\section{Conflicts of Interest}

The Authors have no conflicts of interest in regard to this study.

\section{References}

1 Inoue Y, Hasegawa K, Ishizawa T, Aoki T, Sano K, Beck Y, Imamura $\mathrm{H}$, Sugawara Y, Kokudo N and Makuuchi M: Is there any difference in survival according to the portal tumor thrombectomy method in patients with hepatocellular carcinoma? Surgery 145: 9-19, 2009.

2 Wu CC, Hsieh SR, Chen JT, Ho WL, Lin MC, Yeh DC, Liu TJ and P'eng FK: An appraisal of liver and portal vein resection for hepatocellular carcinoma with tumor thrombi extending to portal bifurcation. Arch Surg 135: 1273-279, 2000.

3 Fukumoto T, Kido M, Takebe A, Tanaka M, Kinoshita H, Kuramitsu K, Komatsu S, Tsugawa D, Goto T, Asari S, Toyama $\mathrm{H}$, Ajiki T and $\mathrm{Ku} \mathrm{Y}$ : New macroscopic classification and backflow thrombectomy for advanced hepatocellular carcinoma with portal vein tumor thrombus invading the contralateral second portal branch. Surg Today 47: 1094-1103, 2017.

4 Yoshiya S, Shirabe K, Nakagawara H, Soejima Y, Yoshizumi T, Ikegami T, Yamashita Y, Harimoto N, Nishie A, Yamanaka T and Maehara Y: Portal vein thrombosis after hepatectomy. World J Surg 38: 1491-1497, 2014.

5 Yamashita Y, Bekki Y, Imai D, Ikegami T, Yoshizumi T, Ikeda T, Kawanaka H, Nishie A, Shirabe K and Maehara Y: Efficacy of postoperative anticoagulation therapy with enoxaparin for portal vein thrombosis after hepatic resection in patients with liver cancer. Thromb Res 134: 826-831, 2014.

6 Liver Cancer Study Group of Japan: The General Rules for the Clinical and Pathological Study of Primary Liver Cancer, Sixth Edition, Revised Version. Tokyo: Kanehara 2015.

7 Soyama A, Eguchi S, Takatsuki M, Hidaka M, Muraoka I, Tomonaga $\mathrm{T}$ and Kanematsu T: Tumor thrombectomy via a surgically reopened umbilical vein combined with right hemihepatectomy in a patient with hepatocellular carcinoma. Dig Surg 28: 222-225, 2011.

8 Hall TC, Garcea G, Metcalfe M, Bilku D and Dennison AR: Management of acute non-cirrhotic and non-malignant portal vein thrombosis: a systematic review. World J Surg 35: 2510-2520, 2011.

9 Rodrigues SG, Maurer MH, Baumgartner I, De Gottardi A and Berzigotti A: Imaging and minimally invasive endovascular therapy in the management of portal vein thrombosis. Abdom Radiol 43(8): 1931-1946, 2018.

Received September 9, 2018

Revised October 17, 2018

Accepted October 23, 2018 